\title{
Knowledge-Based Road Traffic Monitoring
}

\author{
Antonio Fernández-Caballero, Francisco J. Gómez, and Juan López-López \\ Instituto de Investigación en Informática de Albacete (I3A) and \\ Escuela Politécnica Superior de Albacete \\ Universidad de Castilla-La Mancha, 02071-Albacete, España \\ \{caballer,fgomez\}@dsi.uclm.es
}

\begin{abstract}
This article presents a knowledge-based application to study and analyze traffic behavior on major roads, using as the main surveillance artefact a video camera mounted on a relatively high place with a significant image analysis field. The system described presents something new which is the combination of both traditional traffic monitoring systems, that is, monitoring to get information on different traffic parameters and monitoring to detect accidents automatically. Therefore, we present a system in charge of compiling information on different traffic parameters. It also has a surveillance module, which can detect a wide range of the most significant incidents on a freeway or highway.
\end{abstract}

\section{Introduction}

Until a few years ago, surveillance amounted to no more than the presence of policemen on the roads who informed of any road violations. In the last years, there has been a growing interest in the use of automatic mechanisms capable of providing information about the behavior of automobiles on highways and city roads [14 [7 [13. The most attractive of these, without a doubt, is video image detection (e.g. 6] 8] 9]). A survey of video processing techniques for traffic applications [10] demonstrates the great importance of the topic addressed. Notice that every video camera-based traffic control system is classified into two types: (a) Traffic monitoring, which includes: (1) Monitoring to obtain information on different traffic parameters, such as: number of vehicles per unit of time, vehicle classification, average speed, individual speed of each vehicle, etc. (2) Monitoring to detect accidents automatically, also called AID (Automatic Incident Detection). These focus on finding irregularities, such as: stopped traffic, slow traffic, traffic jams, etc. (b) Traffic control for toll purposes or sanctions. The ones focusing on sanctions control traffic violations and detect vehicles unmistakably. Those focusing on tolls analyze traffic at the toll booths, studying the number of vehicles in each line, average waiting time in line, etc.

This article presents an application which allows a study and analysis of traffic behavior on major roads (more specifically freeways and highways), using as the main surveillance artefact a video camera mounted on a relatively high place (such as a bridge) with a significant image analysis field. It is a traffic monitoring system which allows the gathering of some traffic parameters and the detection of some of the most significant and frequent incidents 
that can take place on a freeway or highway. Our approach is similar to [1] in the sense that image-processing modules extract visual data from the scene by spatio-temporal analysis 11 and high-level modules are designed to work on vehicles and their attributes and to exploit expert knowledge on traffic conditions.

\section{Road Traffic Monitoring Architecture}

The system is capable of identifying each vehicle that appears in each image and it assigns them the type of vehicle they belong to and the real situation they occupy on the road. Once a vehicle is detected [12] [2], it is followed closely through the images captured by the video camera, so that each vehicle is tracked down from the time it enters the scene until it leaves it. Through this tracking, we get information from that vehicle regarding traffic parameters, such as speed, number of vehicles of each type that have entered the scene, location and speed of each vehicle, as well as the detection of any incidents on the road. Incident detection is carried out by two modules integrated into the system. These modules of the Road Traffic Monitoring architecture (see Fig. 1) are called Static Analysis and Dynamic Analysis, respectively, depending on how they analyze the current image or the comparison of that image with the previous one. Another feature of the application is letting the user determine which incidents he/she wants to control at all times.

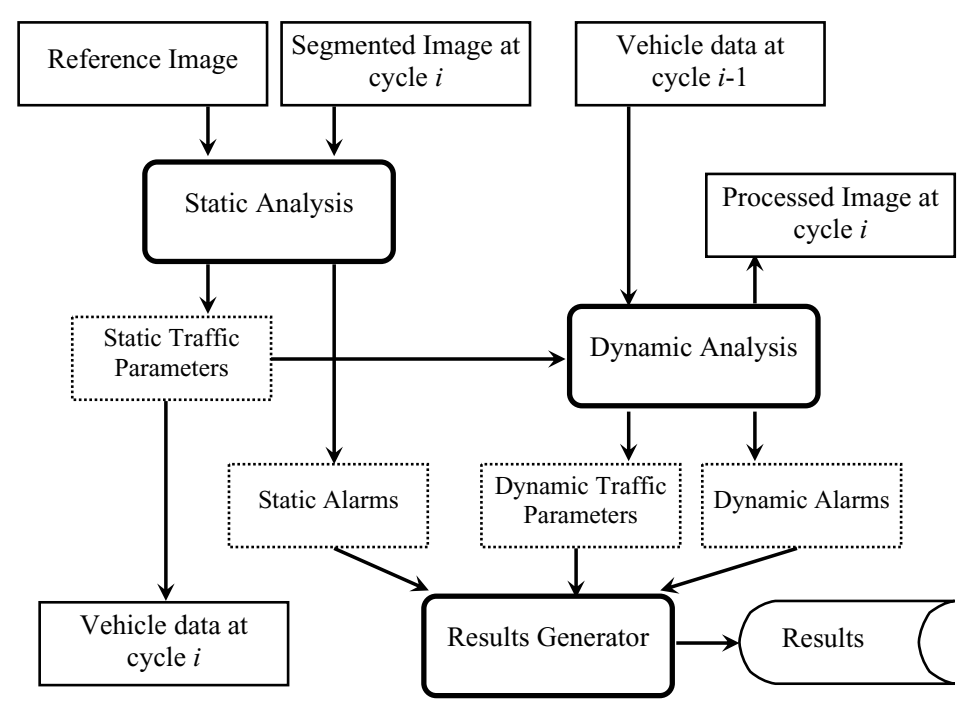

Fig. 1. Road Traffic Monitoring architecture

At the end of the image sequence processing, the system is in the position to offer the following detailed list of parameters: 
- Total number of motorcycles, cars, vans and heavy vehicles (trucks and buses) traveling on the road during the processing period.

- Total number of vehicles traveling in the right and left hand lanes and middle lane, if there is one.

- Total number of vehicles traveling in the entrance/exit ramp, if there is one.

- Total number of vehicles traveling on the road.

- Average speed of traveling motorcycles, cars, vans and heavy vehicles.

- Average speed of vehicles traveling in the right and left hand lanes and middle lane, if there is one.

- Average speed of vehicles traveling in the entrance/exit ramp, if there is one.

- Average speed of all vehicles traveling on the road.

The system uses a camera mounted on a bridge which captures real traffic images. These images, which are processed in a 256-color-gray scale format, are segmented according to 3 , 4] [5] model in such a way that each object's movement captured in the real image may be observed. The segmented image shows the image's background in black, whereas the motion detected in the vehicles is represented by pixels in different gray scales (see Fig. 2b). A great number of pixels can be seen in gray scales which do not represent moving objects; those pixels constitute the image's noise. This application analyzes the sequence of segmented images, giving rise to a sequence of processed images, where each vehicle is shown through an identifying color which is maintained while the vehicle travels along the area of analysis. The shape of the vehicle is rectangular (see Fig. 2r). This whole process is carried out periodically with a 0.5 second frequency.
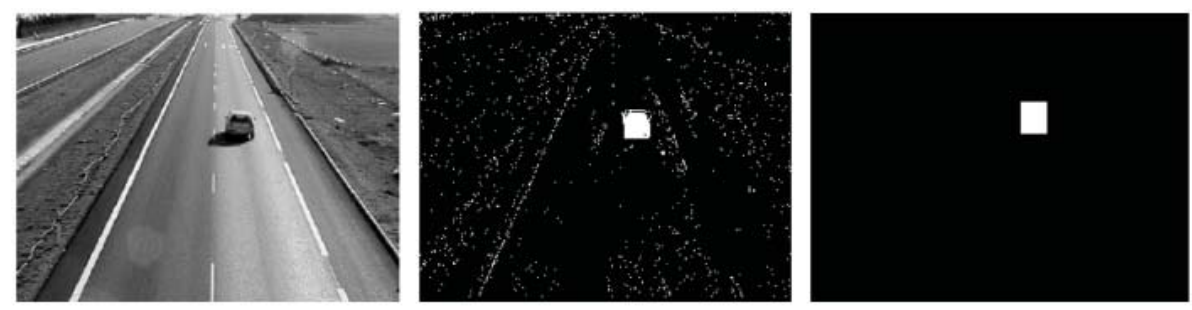

Fig. 2. Road traffic monitoring images. (a) Real image in gray scales. (b) Segmented image. (c) Processed image.

\section{Static Analysis for Road Traffic Monitoring}

Static Analysis uses "Segmented Image at cycle $i$ " and "Reference Image" as inputs and "Static Traffic Parameters" as output. To detect moving objects in the scene, the algorithms described in [5] are performed. Once a moving object has been detected, the dimensions of the object are established, its center is calculated and the coordinates of its center are extrapolated to the "Reference Image", which returns the value of the colors of those coordinates. Those colors 
are related to the position the object occupies, according to Table 3, and are associated to the "Reference Image" (see example in Fig. 3b). Notice that the coordinates of the center of the object are the lower boundary and the half point between the left and right boundaries. This is done this way so that the center can have better correspondence with the real situation of the vehicle.

Table 1. Colors table

\begin{tabular}{lll}
\hline Color & Color (byte) & Area that it represents \\
\hline White & 255 & Reserved \\
Yellow & 252 & Entrance/exit ramp \\
Red & 224 & Shoulder \\
Dark Gray & 146 & Right Lane \\
Light Blue & 31 & Left Lane \\
Green & 28 & Middle Lane \\
Dark Blue & 3 & "Out of bounds" (it does not belong to the road) \\
Black & 0 & Lines and dividing lines \\
\hline
\end{tabular}
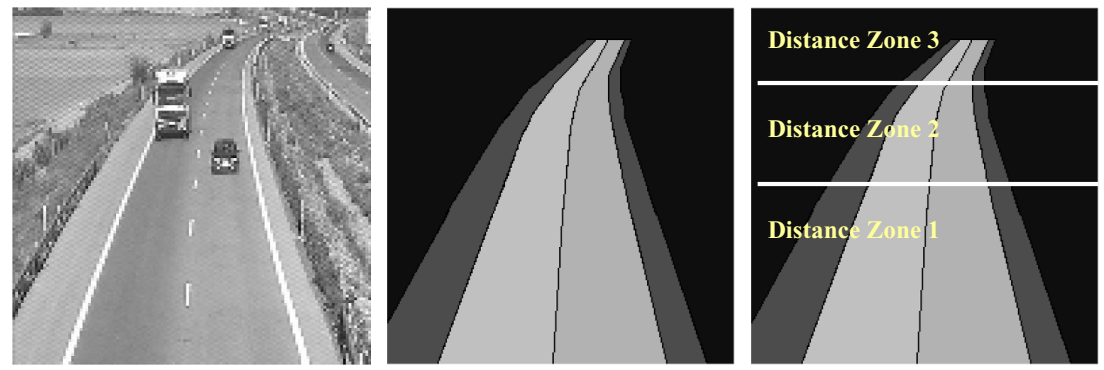

Fig. 3. Example of Reference Image. (a) Road traffic image. (b) Reference Image. (c) Distance zones.

Thus, through moving object detection, the number of moving objects in the area of analysis at the time is determined, as well as their dimensions, and position on the road. A vehicle classification process determines the objects whose established position is not "Out of bounds". A vehicle which inherits the characteristics of the object is created and the object is eliminated. The vehicle is classified, according to its type. The type of vehicles established as valid vehicles are: "Motorcycle", "Car", "Van" or "Heavy Vehicle" (both trucks and buses are included). If the object does not belong to any type, it is labeled as a "Foreign object". Once the vehicle is classified, an estimate of its minimum and maximum position is calculated in the following image, depending on its minimum or maximum speed, respectively. All this information is called "Static Traffic Parameters" and it goes on to the Dynamic Analysis as such and to the following execution cycle under the name of "Vehicle data at cycle $i$ ". 
To know a vehicle's dimensions and the distance between vehicles, three "Distance Zones" have been defined which indicate three areas where the value of the resolution of each pixel, length and crosswise, is going to be the same for that whole area but different from the other areas. The user indicates this area so that the pixels in each distance zone represent a number of centimeters which is lower than the pixels from an area further away from the camera. An example of distance zones for the "Reference Image" is found in Fig. 35.

Now, a static surveillance step is capable of controlling three incidents through the study of the object's/vehicle's dimension and its position, as shown next:

- Vehicle traveling along shoulder: The coordinates in the center of the vehicle are extrapolated to the reference image. Depending on the color of the pixel corresponding to those coordinates, the vehicle will be in one area or another. If the pixel is red, the vehicle is traveling on the shoulder or median.

- Restricted traffic: During those dates when traffic is restricted, the traveling of vehicles in the "heavy vehicles" category is controlled. It is necessary for the user to have previously defined the restriction dates. The task "Static Surveillance" is in charge of comparing those dates with the current date and whether any of the objects detected has "heavy vehicle" characteristics or not.

- Foreign objects: The existence of objects detected that do not belong to any valid type of vehicle is checked.

Hence, the information called "Static Alarms" is generated. For each vehicle detected: (a) the position that the vehicle occupies is checked. If it is on the shoulder, a record is kept informing that the vehicle is traveling on the shoulder; (b) the type of vehicle is checked. If it is a heavy vehicle, the date is checked to see if it is traveling on restricted dates; if it is a "Foreign object", a record is kept indicating that an unidentified object remains in the area of analysis.

\section{Dynamic Analysis for Road Traffic Monitoring}

An image comparison step is the fundamental part of the Dynamic Analysis. It is in charge of finding the differences between an image and the previous one. This is done by comparing the information obtained from the Static Analysis of the vehicles in the current segmented image, located in "Static Traffic Parameters", and information "Vehicle data at cycle $i-1$ ", which contains static information from vehicles in the segmented image included in the previous cycle. The first thing that is calculated is the combinatory relationship of each vehicle in the previous image with each vehicle in the current image. This process is based on weight classification for each relationship, according to the following criteria. It is essential for the current vehicle to be further ahead than the other one, in the direction of traffic. If this is so, then:

- The weight established in parameter Advanced Position is established in the total weight of this relationship. This weight is expressed as $W_{A P}$. 
- If they are in the same lane, the weight established in characteristic Same Lane is added to the total weight of the relationship. The weight for this argument is called $W_{S L}$.

- If both vehicles belong to the same type, the weight established in Same Type is added to the total weight of the relationship. This weight is called $W_{S T}$.

- If the current vehicle is within the position estimates done for the previous vehicle, and it was within the speed limits, the weight established in parameter Within Speed Limits is added to the total weight of the relationship. This weight is called $W_{W S L}$.

If the characteristics of any parameter are not fulfilled, that weight is, naturally, not added to the total weight of the relationship. Therefore, the total weight of a combinatory relationship is called $T W_{C R}$ and it is defined as:

$$
T W_{C R}=W_{A P}+W_{S L}+W_{S T}+W_{W S L}
$$

Once all possible combinatory relationships have been established and the weight of those relationships has been determined, we go on to the final classification between vehicles from the previous and current images. In this process, the combinatory relationships established are compared and a vehicle from the previous image is matched with one from the current image through the selection of the relationship with the greatest possible weight. This is done in such a way that if a match has been established between a previous and a current vehicle and a subsequent relationship containing the same current vehicle with greater weight is found: (a) the previous match is undone, (b) a new match is established for the current vehicle with the previous vehicle from the relationship with the greatest weight, and, (c) another match is tried for the previous vehicle through the search of another relationship which will contain it.

Once this process is completed, the final classifications between vehicles from the previous and current images are obtained. This means that the previous and current vehicles which are matched represent the same real vehicle. There might be previous or current vehicles unable to be matched. This condition can correspond to five different situations, as listed next:

- Current vehicle entering the area of analysis.

- Previous vehicle leaving the area of analysis.

- Previous vehicle which stops on the road after suddenly slowing down.

- Previous vehicle concealed or overlapped by another vehicle.

- Current vehicle coming out of concealment or overlapping or a stopped vehicle that has resumed speed.

When the final matching is done and we know which vehicle from the previous image corresponds to which from the current image, the following operations related to vehicle tracking - are carried out: 
1. A color tag is assigned to the current vehicle, depending on:

(a) if the current vehicle is matched to a previous vehicle, the tag from the previous vehicle is assigned to the current one.

(b) if the current vehicle is not matched to a previous vehicle, it is assigned a new color tag.

2. The vehicle is drawn in the processed image.

Afterwards, the speed of the vehicles is calculated and the traffic parameter counters are updated, according to the vehicles that have entered the scene. For every final match, information about the coordinates of the center of the previous and current vehicles of the match is extracted. The distance traveled by the vehicle is calculated, according to the compromise area where it is situated and where it was situated before, taking into account the resolution of the pixels in the distance zones. Since the distance traveled between two images and the actual period of time between those two images are known, the speed at which the vehicle has been traveling during that time is calculated. The result of all these operations are the "Dynamic Traffic Parameters".

Using the previous information, a dynamic surveillance procedure can start for detecting irregularities linked to object motion. This way information "Dynamic Alarms", which contains all the incidents detected in this execution cycle, is created. That information contains the following items:

- Date and time of the incident.

- Type of incident.

- Type of vehicle involved, as well as the assigned tag.

- Place on the road where the incident has taken place.

The incidents detected by this task, as well as the way to control them, are the following ones:

- Traveling too slowly or too fast: If the speed of the current vehicle exceeds the minimum between the highest speed limit for that type of vehicle and the highest limit for the lane where the vehicle is currently traveling in, an incident involving excessive speed is detected, that is, if the speed is below the maximum between the lowest speed limit for that type of vehicle and the lowest limit for the lane the vehicle is currently traveling in, an incident involving a vehicle traveling too slowly is detected.

- Overstepping a solid line: The reference image is checked to see that there is no solid line established between the position of the previous and current vehicles. To do this, we go through the middle row between the centers of both vehicles and from the column of one of them to that of the other. We also check to see if there are three consecutive black pixels.

- Stopped vehicle: A previous vehicle that is not matched to a current one, that is, a vehicle that has disappeared from the scene and whose speed had slowed down or had traveled at a lower speed that the minimum speed limit.

- Traffic congestion: When the number of stopped vehicles is greater that the threshold set up, it is considered a traffic jam. 
- Strange movement: An object moves in such a way that its transverse movement is greater than its longitudinal one. The longitudinal movement is the number of rows of pixels which separates the previous from the current vehicle. The transverse movement is the number of columns which separates the two.

To this purpose, first the value of the speed of each current vehicle is extracted. If that speed is less than the minimum corresponding speed, an indication that there has been an incident involving lack of speed is stored in a data structure. If the speed is greater than the maximum corresponding limit, information about an incident involving excessive speed is stored. Later, all the final matches are checked over, and:

- The coordinates from the center of the previous and current vehicle implicated in the match are recorded. The longitudinal distance and the transverse length traveled by the vehicle are calculated. If the vehicle's movement is mainly transverse in the scene, an incident involving a strange movement is indicated.

- The segment from the intermediate line which separates the coordinates from both centers is checked over. If three consecutive black pixels are found, an incident of solid line overstepping is reported.

All previous unmatched vehicles are gone through. If any of them is stopped, an incident involving a stopped vehicle is reported. This means that the counter for the number of stopped vehicles increases by one and if that counter is equal to or greater than the threshold established, information about an incident involving a traffic jam is stored.

\section{Data and Results}

Firstly, the performance of the application is studied by taking a controlled vehicle traveling at a constant speed of $100 \mathrm{~km} /$ hour through the area of analysis

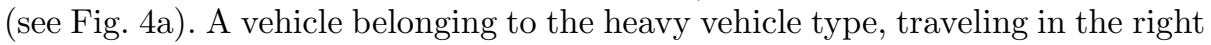
lane at $100 \mathrm{~km} /$ hour, has been detected. The type of vehicle and the lane where it was traveling has been detected correctly. The vehicle has not committed any traffic violations. The next situation is similar to that of the previous example. In this case, the controlled vehicle traveled at a constant speed of $120 \mathrm{~km} /$ hour (see Fig. 4b). It is detected in the right lane traveling at $122 \mathrm{~km} /$ hour. These results practically agree with reality, since the real speed was $120 \mathrm{~km} /$ hour. The deviation in the speed calculation is around $1.6 \%$. As for incidents, the truck is detected to be speeding. The incident is only recorded in the image where it is detected.

In the last study case presented in Fig. 4k, a typical traffic scene with several types of vehicles is analyzed. The weather conditions were partly cloudy with strong winds. Furthermore, the date in which the images were processed is specified as a restricted-traffic date in order to see how the incident detection tool 
works. The traffic parameters obtained in this set are: four vehicles traveling in the right lane at an average speed of $121 \mathrm{~km} /$ hour and only one traveling in the left lane at $129 \mathrm{~km} /$ hour. According to the type of vehicle: three vehicles traveled at an average speed of $141 \mathrm{~km} /$ hour and two heavy vehicles at an average speed of $96 \mathrm{~km} /$ hour. Five vehicles traveled altogether at an average speed of $123 \mathrm{~km} /$ hour. Two heavy vehicles are detected on a restricted date. Moreover, the following vehicles were speeding: the heavy vehicle matched with the yellow one in the processed image, which was traveling in the right lane and the cars matched with the red, gray and blue ones in the processed images.

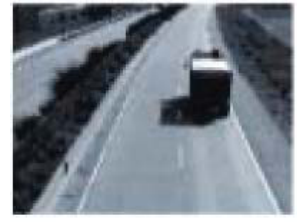

(a1)

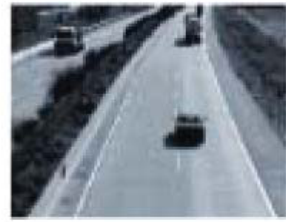

(c1)

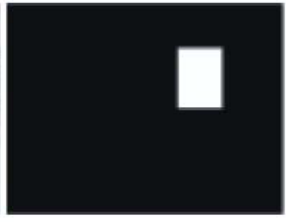

(a2)

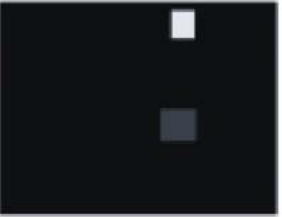

(c2)

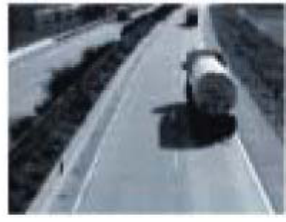

(b1)

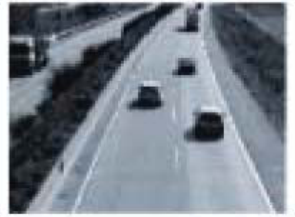

(c3)

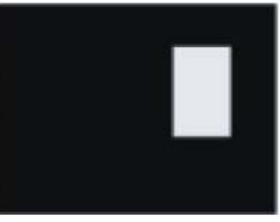

(b2)

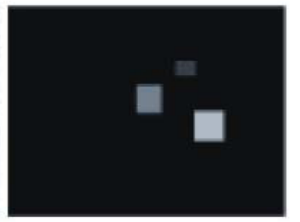

(c4)

Fig. 4. Some road traffic monitoring scenes

\section{Conclusions}

The application proposed implements a knowledge-driven system capable of controlling traffic on highways and freeways in one direction of traffic. At maximum, the application controls three lanes of regular traffic (right, middle and left) and an entrance / exit ramp. The images are captured by a video camera mounted on top of a bridge. Through segmentation, this system provides a sequence of images where the movements of the objects in the scene are shown. The position of each object on the road is calculated and the objects are classified according to the categories of valid type of vehicle or to strange object category. Up to this point in the processing, the application can detect the following traffic incidents: presence of a strange object on the road, heavy vehicle traveling on restricted dates and vehicle traveling on the shoulder.

By analyzing the current and previous segmented images, we can determine the movement of each vehicle in the scene. The application has the capability of matching these vehicles in such a way that vehicles in the previous segmented image are matched to those in the current one. Likewise, by analyzing motion, the application can detect the following incidents: a vehicle speeding or driving 
too slowly, a stopped vehicle, congestion on the road, a vehicle overstepping the solid line and a vehicle's strange movement.

\section{Acknowledgements}

This work is supported in part by the Spanish CICYT TIN2004-07661-C02-02 grant and the Junta de Comunidades de Castilla-La Mancha PBI06-0099 grant.

\section{References}

1. Cucchiara R., Piccardi M., Mello, P. (2000). Image analysis and rule-based reasoning for a traffic monitoringsystem. IEEE Transactions on Intelligent Transportation Systems, 1 (2), 119-130.

2. Fernández M.A., Fernández-Caballero A., López M.T., Mira J. (2003). LengthSpeed Ratio (LSR) as a characteristic for moving elements real-time classification. Real-Time Imaging, 9 (1), 49-59.

3. Fernández-Caballero A., Mira J., Fernández M.A., Lopez M.T. (2001). Segmentation from motion of non-rigid objects by neuronal lateral interaction. Pattern Recognition Letters, 22 (14), 1517-1524.

4. Fernández-Caballero A., Mira J., Delgado A.E., Fernández M.A. (2003). Lateral interaction in accumulative computation: A model for motion detection. Neurocomputing 50, 341-364.

5. Fernández-Caballero, A., Fernández M.A., Mira J., Delgado A.E. (2003). Spatiotemporal shape building from image sequences using lateral interaction in accumulative computation. Pattern Recognition 36 (5), 1131-1142.

6. Gupte S., Masoud O., Martin R.F.K., Papanikiolopoulos N.P. (2002). Detection, and classification of vehicles. IEEE Transactions on Intelligent Transportation Systems, 3 (1), 37-47.

7. Ha D.M., Lee J.M., Kim Y.D. (2004). Neural-edge-based vehicle detection and traffic parameter extraction. Image and Vision Computing, 22 (11), 899-907.

8. Hsieh J.W., Yu S.H., Chen Y.S., Hu W.F. (2006). Automatic traffic surveillance system for vehicle tracking and classification. IEEE Transactions on Intelligent Transportation Systems, 7 (2), 175-187.

9. Ji X., Wei Z., Feng Y. (2006). Effective vehicle detection technique for traffic surveillance systems. Journal of Visual Communication and Image Representation, $17(3), 647-658$.

10. Kastrinaki V., Zervakis M., Kalaitzakis K. (2003). A survey of video processing techniques for traffic applications. Image and Vision Computing, 21 (4), 359-381.

11. López M.T., Fernández-Caballero A., Fernández M.A., Mira J., Delgado A.E. (2006). Visual surveillance by dynamic visual attention method. Pattern Recognition, 39 (11), 2194-2211.

12. Mira J., Delgado A.E., Fernández-Caballero A., Fernández M.A. (2004). Knowledge modelling for the motion detection task: The algorithmic lateral inhibition method. Expert Systems with Applications, 27 (2), 169-185.

13. Rad R., Jamzad M. (2005). Real time classification and tracking of multiple vehicles in highways. Pattern Recognition Letters, 26 (10), 1597-1607.

14. Tai J.C., Tseng S.T., Lin C.P., Song K.T. (2004). Real-time image tracking for automatic traffic monitoring and enforcement applications. Image and Vision Computing, 22 (6), 485-501. 